\title{
Effect of Home-based Prehabilitation in an Enhanced Recovery After Surgery Program for Patients Undergoing Colorectal Cancer Surgery during the COVID-19 Pandemic
}

\section{Francisco López-Rodríguez -Arias}

University General Hospital of Elche: Hospital General Universitario de Elche Luis Sánchez-Guillén ( $\nabla$ drsanchezguillen@gmail.com )

University General Hospital of Elche: Hospital General Universitario de Elche https://orcid.org/00000003-0623-9074

\section{Verónica Aranaz-Ostáriz}

University General Hospital of Elche: Hospital General Universitario de Elche

\section{Daniel Triguero-Cánovas}

University General Hospital of Elche: Hospital General Universitario de Elche

\section{Sandra Lario-Pérez}

University General Hospital of Elche: Hospital General Universitario de Elche

\section{Xavier Barber-Vallés}

University General Hospital of Elche: Hospital General Universitario de Elche

\section{Francisco Javier Lacueva}

University General Hospital of Elche: Hospital General Universitario de Elche

Jose M Ramírez

Lozano Blesa University Clinical Hospital: Hospital Clinico Universitario Lozano Blesa

\section{Antonio Arroyo}

University General Hospital of Elche: Hospital General Universitario de Elche

\section{Research Article}

Keywords: Prehabilitation, COVID-19, confinement, Body composition, Lean Mass and Fat Mass.

Posted Date: May 28th, 2021

DOI: https://doi.org/10.21203/rs.3.rs-309905/v1

License: (a) This work is licensed under a Creative Commons Attribution 4.0 International License. Read Full License 


\section{Abstract}

\section{Background}

Surgery remains the first curative treatment for colorectal cancer. Prehabilitation seems to attenuate the loss of lean mass in the early postoperative period. However, its long-term role has not been studied. Lockdown due to COVID-19 pandemic has forced to carry out the prehabilitation program at home.

This study aimed to assess the effect of home prehabilitation on body composition, complications and hospital stay in patients undergoing oncological colorectal surgery.

\section{Méthods}

A prospective and randomized clinical study was conducted in 20 patients operated of colorectal cancer during COVID-19 lockdown (13th March -21st June 2020) in a single university clinical hospital. Patients was randomly into two study groups (10 per group): Prehabilitation vs Standard Care. Changes in lean mass and fat mass at 45 and 90 days after surgery was measured using multifrequency bioelectrical impedance analysis.

\section{Results}

Prehabilitation managed to reduce hospital stay (4.8 vs 7.2 days, $p=0.052)$ and postoperative complications $(20 \%$ vs $50 \%, p=0.16)$.

45 days after surgery, the loss of lean mass decreased $(1.7 \%$ vs $7.1 \%, p=0.17)$. These differences in lean mass were attenuated at 90 days, however, the standard care group increased considerably their fat mass compared to the prehabilitation group ( $+8.72 \%$ vs $-8.16 \%)$.

\section{Conclusions}

Home prehabilitation has proven its effectiveness achieving an attenuation of lean mass loss in the early postoperative period and a lower gain in fat mass in the late postoperative period. In addition, it has managed to reduce hospital stay and postoperative complications.

\section{Registration number}

This article is part of an ongoing, randomized and controlled clinical trial approved by the Ethics Committee of our Hospital and registred in ClinicalTrials.gov in August 2018 with registration number NCT03618329.

\section{Introduction}

Colorectal cancer (CRC) remains the third most common cancer diagnosis in Western countries, and tumor resection and regional lymphadenectomy form the basis of its treatment. ${ }^{1,2}$ In more advanced 
stages and for tumors with poor prognostic factors, adjuvant chemotherapy in recommended cases has been shown to decrease recurrence and improve survival. ${ }^{3-6}$

However, many patient-dependent factors, such as malnutrition, sarcopenia or a deterioration in general status, have been associated with poorer perioperative outcomes and a delay in the start of adjuvant chemotherapy, with globally worse oncological outcomes being obtained. ${ }^{7-10}$ Adequate nutritional status with preservation of lean mass (LM) has become an important objective to be considered in the perioperative period of patients with CRC..$^{11-14}$

Prehabilitation aims to optimize the patient's health during the period between diagnosis and surgery, in order to reduce complications derived from surgery, thereby promoting an early recuperation of the patient's baseline condition. This is achieved by improving physical condition, optimizing nutritional status, and acting at the cognitive level to try to reduce stress and anxiety levels. ${ }^{15,16}$ Perioperative prehabilitation before and after surgery appears to attenuate LM loss in the early postoperative period, at 4-8 weeks. However, its long-term effect has not been studied to date. ${ }^{17}$

The COVID-19 pandemic has forced multiple countries worldwide to take exceptional measures such as limiting free movement of people and instating mandatory home confinement, restricting any out-ofhome movements to purposes deemed essential; in our case, all prehabilitation had to be completed at home. After the period of peak COVID-19 prevalence, countries adopted de-escalation policies with a phased easing of measures, in some cases allowing people to leave their homes for daily physical exercise.

The primary objective of this study is to assess the impact of prehabilitation on the body composition of patients undergoing colorectal surgery enrolled in a home-based prehabilitation program versus Standard of Care (Enhanced Recovery After Surgery (ERAS) without prehabilitation) and its impact on postoperative outcomes in the home confinement context due to the COVID-19 pandemic.

\section{Methods}

This study is part of an ongoing randomized, controlled clinical trial with two study groups, the Prehabilitation (PH) group and the Standard of Care (SC) group (ERAS without prehabilitation). We analyzed a cohort of 20 patients affected by the consequences of COVID-19 pandemic, both in the Prehabilitation intervention group and the Standard of Care group.

Patients undergoing elective surgery for colon or rectal neoplasm during the mandatory home confinement period instated by Spanish government authorities from 13 March to 21 June 2020, were included consecutively. Patients with metastatic disease or nutritional supplementation at diagnosis and/or chemotherapy-radiotherapy prior to surgery were excluded. In addition, a minimum physical condition and/or autonomy allowing the patient to safely perform the program exercises was required. 
The sample size was calculated to compare the incidence of complications postoperative in the control group (SC) versus to the intervention group (PH). With a confidence level of $95 \%$ (alpha=0.05) and power of $80 \%$ (Beta=0.2) in a bilateral contrast, 11 subjects are required in the first group and 11 in the second to detect as statistically significant the difference between two proportions, wich for the SC group is expected to be 0.35 and the $\mathrm{PH}$ group from 0.17 , assuming $10 \%$ of losses.

During the preoperative consultation 24 patients were assessed for eligibility. The 20 patients who met the inclusion criteria were given the option to be included in the clinical trial, informed consent was obtained, and randomized into one of two groups: the SC group that followed the standardized ERAS perioperative care protocols, and the $\mathrm{PH}$ group, to which a prehabilitation intervention protocol was added. ${ }^{18}$ Block randomization was carried out by random sequence obtained previously. The patients in both groups underwent minimally invasive surgery performed by members of the colorectal surgery unit.

The primary study variables were changes in patient weight, LM and fat mass (FM). These measurements were taken in both groups at diagnosis, the day before surgery and 6 and 12 weeks after surgery using multifrequency bioelectrical impedance analysis with the Tanita ${ }^{\mathrm{O}} \mathrm{MC} 780$ device. Patients who received adjuvant chemotherapy treatment were excluded from the 12-week postoperative measurement. In addition, two criteria were established to determine whether the patient had suffered a deterioration in body composition: loss of LM greater than $2 \%$, and patients who had not yet presented significant changes in their $\mathrm{LM}( \pm 1 \%$ change) but who experienced a gain in FM of more than $2 \%$.

At the cognitive level, to assess patient anxiety and/or depression levels, determinations were made using a validated version of the Hospital Anxiety and Depression Scale (HADS). ${ }^{19}$

The clinical variables analyzed included age, gender, body mass index (BMI), major comorbidity, American Society of Anesthesiologists-Physical Status (ASA-PS) score, type of surgery, tumor stage, and use of anxiolytic and/or antidepressant medication. Time of hospital stay and postoperative complications occurring within the first 30 days were collected and divided into minor (classified as Clavien-Dindo I-II), which included low-risk events such as surgical wound infection or postoperative ileus, and major (Clavien-Dindo III-IV), which included life-threatening events and cases requiring radiological, surgical or endoscopic interventions to resolve them, such as anastomotic leaks, intra-abdominal collections, or pneumonia. ${ }^{20}$

This study obtained the approval from the Ethics Committee of our hospital with registration number NCT03618329.

\section{Trimodal prehabilitation interventions adapted to the COVID-19 pandemic situation.}

The prehabilitation program was trimodal, with recommendations on physical exercise, nutritional supplementation and relaxation exercises to be performed at home for 30 days before surgery and the 
first 30 days after hospital discharge.

The Canadian Study of Health and Aging Clinical Frailty Scale allowed us to subjectively assess the physical condition of the patient and personalize the type of physical exercise program to be performed and its objectives. ${ }^{21}$ The program was adapted so that patients could complete it at home every day using a video playlist with an approximate duration of 30-45 minutes. The videos included a combination of aerobic and muscular resistance training.

Nutritionally, all patients received dietary recommendations, highlighting the limitation of calorie intake so as not to gain weight and the reduction of toxic habits. In addition, high-protein nutritional supplementation, with high vitamin D and calcium- $\beta$-hydroxy- $\beta$-methylbutyrate (CaHMB) content, (Ensure Plus Advance, Abbott $\AA$ ) was administered to guarantee a minimum supply of 1.2 to $1.5 \mathrm{~g}$ of protein/kg/day.

In order to reduce perioperative anxiety, at the time of diagnosis, all patients received recommendations for relaxation and breathing exercises to be performed at least twice a week.

Patients in the SC group who agreed to participate in the study did not receive any education or recommendation on guidelines for physical activity, nutrition or relaxation, according to standard clinical practice.

\section{Statistical analysis.}

Data analysis was performed by comparing the Prehabilitation group to the control group using IBM SPSS Statistics software.

All continuous variables were analyzed using a t-test or Mann-Whitney $\mathrm{U}$ test from independent samples. All categories were described as percentages and were compared using a Chi-squared test or Fisher's exact test.

P-values $<0.05$ were considered significant.

\section{Results}

A total of 20 patients were included, 10 in the $\mathrm{PH}$ group and 10 in the SC group; $65 \%$ of them were males, with a median age of 65.5 years $(S D=9.2)$.

Table 1 presents the demographic data on age, gender, anthropometric parameters, comorbidities, ASA classification for anesthesia, tumor stage, type of surgery performed and postoperative complications. No statistically significant differences were found between the two study groups. 
Table 1

Characteristics of patients in the study cohort and both groups.

\begin{tabular}{|c|c|c|c|}
\hline & Study cohort $(n=20)$ & $\begin{array}{l}\text { Prehabilitation }(n= \\
\text { 10) }\end{array}$ & $\begin{array}{l}\text { Standard care }(n= \\
\text { 10) }\end{array}$ \\
\hline Age median (years) & $\begin{array}{l}66(61,8-71,5) \mathrm{SD}= \\
9,4\end{array}$ & $\begin{array}{l}66,5(57,7-70) S D= \\
10,2\end{array}$ & $\begin{array}{l}66(64,7-75,5) S D= \\
8\end{array}$ \\
\hline Sex ratio (F:M) & $7: 13(35 \%-65 \%)$ & $4: 6(40 \%-60 \%)$ & 3:7 (30\%-70\%) \\
\hline $\begin{array}{l}\text { Body mass index median } \\
\left(\mathrm{Kg} / \mathrm{m}^{2}\right)\end{array}$ & $\begin{array}{l}26,8(24,5-28,8) S D \\
=4,3\end{array}$ & $\begin{array}{l}27,5(23,2,-30,9) S D \\
=5,6\end{array}$ & $\begin{array}{l}25,8(24,7-28,2) S D \\
=2,8\end{array}$ \\
\hline \multicolumn{4}{|l|}{ ASA } \\
\hline 1 & $5(25 \%)$ & $3(60 \%)$ & $2(40 \%)$ \\
\hline 2 & $9(45 \%)$ & $5(56 \%)$ & $4(44 \%)$ \\
\hline 3 & $6(30 \%)$ & $2(33 \%)$ & $4(67 \%)$ \\
\hline \multicolumn{4}{|l|}{ Co-morbility } \\
\hline Diabetes & $3(15 \%)$ & $2(67 \%)$ & $1(33 \%)$ \\
\hline Smoker & $6(30 \%)$ & $2(33 \%)$ & $4(67 \%)$ \\
\hline Hypertension (HBP) & $5(25 \%)$ & $2(40 \%)$ & $3(60 \%)$ \\
\hline \multicolumn{4}{|l|}{ Type of surgery } \\
\hline Right hemicolectomy & $9(45 \%)$ & $3(33,3 \%)$ & $6(66,7 \%)$ \\
\hline Left hemicolectomy & $1(5 \%)$ & 1 & 0 \\
\hline Sigmoidectomy & $5(25 \%)$ & $4(80 \%)$ & $1(20 \%)$ \\
\hline Low anterior resection & $5(25 \%)$ & $2(40 \%)$ & $3(60 \%)$ \\
\hline \multicolumn{4}{|l|}{ TNM staging system } \\
\hline T0-T1-Tis & $12(60 \%)$ & $7(58,3 \%)$ & $5(41,7 \%)$ \\
\hline T2-T3 & $7(35 \%)$ & $3(42,9 \%)$ & $4(57,1 \%)$ \\
\hline T4 & $1(5 \%)$ & 0 & 1 \\
\hline NO & $15(75 \%)$ & $8(53,3 \%)$ & $7(46,7 \%)$ \\
\hline N1 & $5(25 \%)$ & $2(40 \%)$ & $3(60 \%)$ \\
\hline Adjuvant hemotherapy & $5(25 \%)$ & $2(40 \%)$ & $3(60 \%)$ \\
\hline $\begin{array}{l}\text { Anxiolytic or depression } \\
\text { treatment }\end{array}$ & $6(30 \%)$ & $3(50 \%)$ & $3(50 \%)$ \\
\hline
\end{tabular}




\begin{tabular}{|llll|}
\hline & Study cohort $(\mathrm{n}=\mathbf{2 0})$ & $\begin{array}{l}\text { Prehabilitation }(\mathrm{n}= \\
10)\end{array}$ & $\begin{array}{l}\text { Standard care }(\mathrm{n}= \\
10)\end{array}$ \\
\hline Global complications & $7(35 \%)$ & $2(20 \%)$ & $5(50 \%)$ \\
\hline Surgical site infections & $3(43 \%)$ & 0 & $3(30 \%)$ \\
\hline Other complications & $4(57 \%)$ & $2(20 \%)$ & $2(20 \%)$ \\
\hline
\end{tabular}

The mean duration of the prehabilitation period before surgery was 28.9 days $(S D=2.8)$. The postoperative follow-up of the patients in the short and long term occurred at 44.6 days $(S D=4.2)$ and at 90.6 days $(S D=6.9)$.

A total of $70 \%$ of PH patients experienced loss of LM before surgery, with a mean loss of $1.29 \%$ (SD = 4.2). However, in the early postoperative period, 45 days after surgery, LM loss was minimal. On comparing the $\mathrm{PH}$ patients with those in the SC group, the loss of LM from the time of diagnosis was $1.7 \%(S D=2.32)$ versus $7.1 \%(S D=7.7)$ in the SC group $(p=0.17)$. In addition, prehabilitation was shown to attenuate the deterioration of body composition as compared to the control group ( $20 \%$ vs $80 \%$ ), according to the criteria, 45 days after surgery $(p=0.001)$.

On the other hand, the differences in LM were attenuated in the late postoperative period. At 90 days postsurgery, the PH group had an increase in LM from diagnosis of $+0.15 \%(S D=2.33)$ versus $+1.45 \%(S D=$ $6.23)$ in the SC group. However, while in the SC group FM considerably increased by $8.72 \%(S D=20.03)$, in the $\mathrm{PH}$ group, it decreased by $8.16 \%(\mathrm{SD}=15.09)$. With regard to body weight, patients in the $\mathrm{PH}$ group lost a mean of $1.46 \mathrm{~kg}(S D=4.7)$ while those in the $S C$ group gained $1.6 \mathrm{~kg}(S D=2.97)$.

In the comparative analysis between the two groups, hospital stay was shorter in patients in the $\mathrm{PH}$ group, 4.8 days $(S D=1)$ versus 7.2 days $(S D=3.2)$ in the $S C$ group $(p=0.052)$. Postoperative complications were also lower in PH patients $(20 \%$ vs $50 \%, p=0.16)$. In the SC group three patients had surgical site infections, one patient ileus and another one pneumonia. Complications in the PH group were one lower $\mathrm{GI}$ bleeding in the late postoperative period and one surgical wound bleeding.

No patient presented an anastomotic leak.

The means and standard deviations of the scores obtained in HADS-A and HADS-D are shown in Table 2.

Table 2

Mean score and standard desviation of HADS-A and HADS-D.

\begin{tabular}{|lllll|}
\hline & Diagnosis & Surgery & 45 days postoperative & 90 days postoperative \\
\hline HADS-A & $6,4 \mathrm{SD}=3,5$ & $6,6 \mathrm{SD}=3,9$ & $6,1 \mathrm{SD}=2,5$ & $5,3 \mathrm{SD}=4,36$ \\
HADS-D & $3,6 \mathrm{SD}=4,2$ & $3,4 \mathrm{SD}=4,2$ & $4,1 \mathrm{SD}=1,9$ & $4 \mathrm{SD}=4$ \\
\hline
\end{tabular}


In the anxiety sphere (HADS-A), it was found that $35 \%$ of patients had a pathological score at diagnosis, increasing to $40 \%$ at the time of surgery. During the postoperative period, these figures decreased from $30 \%$ at 45 days of follow-up to $25 \%$ at 90 days.

In the depression sphere (HADS-D), $15 \%$ of patients had a probably pathological score at diagnosis. These values remained stable at the time of surgery, as well as during the different postoperative measurements.

It was found that $30 \%$ of the patients in our sample were chronically taking some type of anxiolytic or antidepressant treatment before diagnosis. These same patients had higher HADS-A scores both at diagnosis $(p=0.03)$ and on the day of surgery $(p=0.07)$. Higher scores were also seen in the HADS-D questionnaire at long-term follow-up $(p=0.02)$.

Finally, patients who underwent adjuvant chemotherapy had higher values in both the HADS-A and HADSD questionnaires in the early postoperative period $(p=0.02)$.

\section{Discussion}

To the best of our knowledge, this is the first project to evaluate the impact of outpatient prehabilitation during the mandatory home isolation period due to the COVID-19 health crisis.

As our results show, in terms of body composition, prehabilitation attenuates LM loss in the early postoperative period, $4-8$ weeks after surgery. ${ }^{16}$ This could have a significant clinical and prognostic significance in prehabilitated patients, because a delay in the start of chemotherapy treatment beyond 8 weeks after surgery has been shown to have poorer oncological outcomes. ${ }^{7,8}$

Moreover, loss of LM during adjuvant CT treatment in patients with metastatic CRC has been related to a poorer tolerance and a poorer response to treatment. Thus, any measure that results in a reduction in loss of LM will ensure greater treatment efficacy and a better long-term prognosis. ${ }^{22,23}$

Furthermore, in our patients, prehabilitation had a protective effect on body composition during the period of immobility caused by the mandatory home confinement due to COVID-19, stabilizing weight gain and FM in long-term follow-up. Therefore, we have found that prehabilitated patients achieved better physical fitness with a faster recovery of their baseline activities of daily living after the end of the confinement period.

Bioelectrical impedance analysis makes it possible to calculate body composition by measuring the resistance to an electric current passing through the body. It is a valid, reliable, simple, inexpensive and safe method to assess fat-free mass in patients with non-metastatic CRC, and its use to evaluate 
changes in body composition in prehabilitation programs has already been studied with good results. ${ }^{24-}$ 26

Prehabilitation in CRC surgery has helped improve the outcomes of ERAS protocols. The respective metaanalyses of Gillis et al. and Bolshinsky et al. showed that prehabilitation achieves a reduction in hospital stay by 2 days and a decrease in the rate of complications derived from surgery, outcomes that are similar to the data obtained in our study. ${ }^{27,28}$

The lack of statistical significance is related to the small sample size, because the observation period or home confinement period was very short. However, the results of our study are promising and show the clinical importance of complying with the ERAS protocols and of systematically including prehabilitation in them, even if performed at home in times of movement restrictions or limitations.

Our study found that both diagnosis and surgery were life situations that induce high levels of stress. The mean HADS-A scores were higher than those obtained postoperatively, consistent with the published scientific evidence ${ }^{29}$.

There are studies reflecting that between $25 \%$ and $50 \%$ of cancer patients present symptoms of depression during treatment. ${ }^{30}$ Satin et al concluded that cancer patients suffering from symptoms of depression had an increase in mortality rate of $26 \%$, and for those with major depression, the mortality rate increased to $39 \% .{ }^{31}$ All of this underscores the importance of early detection of patients with symptoms of anxiety-depression and those most likely to suffer from them, of the implementation of preventive measures to avoid them, as well as of the importance of the implementation of therapeutic strategies to alleviate symptoms already present. Along these lines, we found that patients taking anxiolytic or antidepressant treatments prior to diagnosis had higher HADS-A values during the perioperative period. These patients would have possibly benefited from individualized treatment. In fact, there is evidence that in-person sessions with scheduled home exercises for self-control of symptoms achieve a significant reduction in those symptoms. ${ }^{32}$

In addition, we found that there are other critical moments that can affect the emotional sphere of the patient, such as the need for postoperative adjuvant chemotherapy.

The follow-up of ERAS protocols results in shorter hospital stays and a lower incidence of postoperative complications, which is particularly valuable during the COVID-19 pandemic, where the availability of regular admission hospital beds and intensive care beds has been indispensable for the management of the healthcare crisis. ${ }^{33-35}$ Optimizing these resources during the pandemic allows us to perform cancer surgeries without excessive delays; said delays would lead to more advanced disease staging, together with a delay in the start of adjuvant chemotherapy, which in turn would result in poorer overall oncological outcomes. Similarly, the reduction in length of hospital stay has contributed to decreased exposure to the risk of in-hospital contagion of SARS-CoV-2, a perioperative nosocomial infection of which could have dire clinical consequences. ${ }^{36}$ The COVID-19 era has forced us to change our diagnostic 
and treatment strategies for patients with CRC. ${ }^{37}$ Our study has shown that it is possible to carry out prehabilitation programs at home, achieving satisfactory results both in terms of preservation of patient body composition and in reducing complications and length of hospital stay.

As limitations of our work, we must highlight the low sample size, explained by the short observational period because of the duration of home-based confinement dictated by government authorities.

\section{Conclusion}

Home-based prehabilitation has shown to be effective, achieving an attenuation of loss of LM in the early postoperative period and decreasing the FM in the late postoperative period. In addition, its inclusion in ERAS protocols has reduced length of hospital stay and perioperative complications helping to fight against the health collapse caused by the COVID-19 pandemic.

In select cases, even in lockdown situations, prehabilitation can be carried out on an outpatient basis. More studies with a larger sample of patients will be necessary to confirm the benefits obtained in our study. Furthermore, outstanding issues as the cost-effective analysis of prehabilitation programs, the need to adapt and individualize these programs to the physical condition and body composition of patients, or the use of new technologies to monitor prerehabilitation programs.

\section{Declarations}

-Funding: This research did not receive any specific grant from funding agencies in the public, commercial, or not-for-profit sectors.

-Conflicts of interest/Competing interests: The authors declare that they have no conflict of interest.

-Availability of data and material: The datasets generated during and/or analysed during the current study are not publicly available due because de limitations of the law of data protections of our country but are available from the corresponding author on reasonable request.

\section{-Code availability: N/A}

\section{-Author contributions}

Francisco López-Rodríguez-Arias: Conceptualization, investigation and writing - Original Draft. Luis Sánchez-Guillén: Conceptualization, Writing -Review \& Editing and Visualization. Verónica Aranaz Ostáriz: Writing -Review \& Editing and investigation. Daniel Triguero: Investigation. Cánovas Sandra Lario Perez: Investigation. Xavier Barber: Formal analysis. Francisco J Lacueva: Writing -Review \& Editing and Supervisión. José M Ramírez: Supervision. Antonio Arroyo: Conceptualization, Writing -Review \& Editing and Project adminsitration.

-Ethics approval: Approval was obteined from the ethics committee of our hospital. 
-Consent to participate: Informed consent was obtained from all individual participants included in the study.

-Consent to publication: The patients were informed that the data obtainedduring the study could be used for publications in scientific journals.

-Acknowledgements: We thank all the participants and the doctors who contributed to this project.

\section{References}

1. Lamanna A, Sheaffer H, Guerra C, Kochman M (2016). Colorectal cancer screening navigation for the underserved: experience of an urban program. Gastroenterol Hepatol ( $N$ Y ) 12:547-551.

2. Figueredo A, Rumble RB, Maroun J, et al (2003) Gastrointestinal Cancer Disease Site Group of Cancer Care Ontario's Program in Evidence-based Care. Follow-up of patients with curatively resected colorectal cancer: a practice guideline. BMC Cancer 3:26. https://doi.org/10.1186/1471-2407-3-26

3. Vogel JD, Eskicioglu C, Weiser MR, Feingold DL, Steele SR (2017) The American Society of Colon and Rectal Surgeons clinical practice guidelines for the treatment of colon cancer. Dis Colon Rectum 60:999-1017. https://doi.org/10.1097/DCR.0000000000000926.

4. Benson AB, Venook AP, Al-Hawary MM, et al (2018) NCCN guide- lines insights: colon cancer, version 2.2018. J Nat/ Compr Canc Netw 16:359-369. https://doi.org/10.6004/jnccn.2018.0021.

5. André T, Boni C, Navarro M, et al (2009) Improved overall survival with oxaliplatin, fluorouracil, and leucovorin as adjuvant treatment in stage II or III colon cancer in the MOSAIC trial. J Clin Oncol 27:3109-3116. https://doi.org/10.1200/JC0.2008.20.6771.

6. Grothey A, Sobrero AF, Shields AF, et al (2018) Duration of adjuvant chemotherapy for stage III colon cancer. N Engl J Med 378:1177-1188. https://doi.org/10.1056/NEJMoa1713709.

7. Kim IY, Kim BR, Kim YW (2015) Factors affecting use and delay ( $\geq 8$ weeks) of adjuvant chemotherapy after colorectal cancer surgery and the impact of chemotherapy-use and delay on oncologic outcomes. PLoS On 10:e0138720. https://doi.org/10.1371/journal.pone.0138720.

8. Biagi JJ, Raphael MJ, Mackillop WJ, Kong W, King WD, Booth CM (2011) Association between time to initiation of adjuvant chemotherapy and survival in colorectal cancer: a systematic review and meta-analysis. JAMA 305:2335-2342. https://doi.org/10.1001/jama.2011.749.

9. Malietzis G, Aziz O, Bagnall NM, Johns N, Fearon KC, Jenkins JT (2015) The role of body composition evaluation by computerized tomography in determining colorectal cancer treatment outcomes: a systematic review. Eur J Surg Oncol 41:186-96.

https://doi.org/10.1016/j.ejso.2014.10.056.

10. Malietzis G, Currie AC, Athanasiou T, Johns N, Anyamene N, Glynne-Jones R, et al (2016) Influence of body composition profile on outcomes following colorectal cancer surgery. Br J Surg 103:572-80. https://doi.org/10.1002/bjs.10075. 
11. Tsai S (2012) Importance of lean body mass in the oncologic patient. Nutr Clin Pract 27:593-8. https://doi.org/10.1177/0884533612457949.

12. Baracos VE (2015) Skeletal muscle anabolism in patients with advanced cancer. Lancet Oncol 16:13e4. https://doi.org/1016/S1470-2045(14)71185-4.

13. Trejo-Avila M, Bozada-Gutiérrez K, Valenzuela-Salazar C, Herrera-Esquivel J, Moreno-Portillo M (2021) Sarcopenia predicts worse postoperative outcomes and decreased survival rates in patients with colorectal cancer: a systematic review and meta-analysis. Int J Colorectal Dis. 36:1077-1096. https://doi.org/10.1007/s00384-021-03839-4.

14. Ryan AM, Prado CM, Sullivan ES, Power DG, Daly LE (2019) Effects of weight loss and sarcopenia on response to chemotherapy, quality of life, and survival. Nutrition. 67-68:110539. https://doi.org/10.1016/j.nut.2019.06.020.

15. Gillis C, Buhler K, Bresee L, Carli F, Gramlich L, Culos-Reed N, et al (2008) Effects of nutritional prehabilitation with and without exercise, on outcomes of patients who undergo colorectal surgery: A systematic review and meta-analysis. Gastroenterology 155:391-410. http://doi.org/10.1053/j.gastro.2018.05.012.

16. López Rodríguez-Arias F, Sánchez-Guillén L, Armañanzas Ruiz LI et al (2020) A Narrative Review About Prehabilitation in Surgery: Current Situation and Future Perspectives. Revisión narrativa de la prehabilitación en cirugía: situación actual y perspectivas futuras. Cir Esp. 98:178-186. http//doi.org/10.1016/j.ciresp.2019.11.005.

17. Gillis C, Fenton TR, Sajobi TT, et al (2019) Trimodal prehabilitation for colorectal surgery attenuates post-surgical losses in lean body mass: A pooled analysis of randomized controlled trials. Clin Nutr 38:1053-1060. http//doi.org/10.1016/j.clnu.2018.06.982.

18. https://www.grupogerm.es/protocolos-zaragoza.

19. Snaith RP, Zigmond AS (1986) The hospital anxiety and depression scale. Br Med J 292: 344. http//doi.org/10.1136/bmj.292.6516.344.

20. Clavien PA, Barkun J, de Oliveira ML, et al (2009) The Clavien-Dindo classification of surgical complications: five-year experience. Ann Surg 250:187-196. http//doi.org/10.1097/SLA.0b013e3181b13ca2.

21. Rockwood K, Song X, MacKnight C, et al (2005) A global clinical measure of fitness and frailty in elderly people. CMAJ. 173:489-495. http//doi.org/10.1503/cmaj.050051.

22. Hassinger TE, Mehaffey JH, Martin AN et al (2019) Implementation of an Enhanced Recovery Protocol is Associated With On-Time Initation of Adjuvant Chemotherapy in Colorectal Cancer. Dis Colon Rectum. 62:1305-1315. http//doi.org/10.1097/DCR.0000000000001486.

23. Sasaki S, Oki E, Saeki H, et al (2019) Skeletal muscle loss during systemic Chemotherapy for colorectal cancer indicates treatment response: a pooled analysis of a multicenter clinical trial (KSCC 1605-A) Int J Clin Oncol 24:1204-1213. http//doi.org/10.1007/s10147-019-01460-8.

24. Ræder H, Kværner AS, Henriksen C, et al (2018) Validity of bioelectrical impedance analysis in estimation of fat-free mass in colorectal cancer patients. Clin Nutr37:292-300. 
http//doi.org/10.1016/j.clnu.2016.12.028.

25. Mulasi U, Kuchnia AJ, Cole AJ, Earthman CP (2015) Bioimpedance at the bedside: current applications, limitations, and opportunities. Nutr Clin Pract 30:180-

193. http//doi.org/10.1177/0884533614568155. (Erratum in: Nutr Clin Pract. 2015 30:589).

26. Gillis C, Fenton TR, Sajobi TT, et al (2019) Trimodal prehabilitation for colorectal surgery attenuates post-surgical losses in lean body mass: A pooled analysis of randomized controlled trials. Clin Nutr 38:1053-1060. http//doi.org/10.1016/j.clnu.2018.06.982

27. Gillis C, Buhler K, Bresee L, et al (2018) Effects of Nutritional Prehabilitation, With and Without Exercise, on Outcomes of Patients Who Undergo Colorectal Surgery: A Systematic Review and Metaanalysis. Gastroenterology 155:391-410.e4. http//doi.org/10.1053/j.gastro.2018.05.012.

28. Bolshinsky V, Li MH, Ismail H, Burbury K, Riedel B, Heriot A (2018) Multimodal Prehabilitation Programs as a Bundle of Care in Gastrointestinal Cancer Surgery: A Systematic Review. Dis Colon Rectum 61:124-138. http//doi.org/10.1097/DCR.0000000000000987.

29. Parle $M$, Jones $B$, Maguire $P$ (1996) Maladaptive coping and affective disorders among cancer patients. Psychol Med 26: 735- 744. http//doi.org/10.1017/s0033291700037752.

30. Schwarz R, Krauß O (2000) Palliative medicine-psychological therapy. Internist 7:612-8. http//doi.org/10.1007/s001080050578.

31. Satin JR, Linden W, Phillips MJ (2009) Depression as a predictor of disease progression and mortality in cancer patients. Cancer 115:5349-61. http//doi.org/10.1002/cncr.24561.

32. Li J, Liu X (2019) Incremental patient care program decreases anxiety, reduces depression and improves the quality of life in patients with colorectal cancer receiving adjuvant chemotherapy. Exp Ther Med 18:2789-2798Exp Ther Med 18:2789-2798. http//doi.org/10.3892/etm.2019.7877.

33. Borghi F, Pellegrino L, Pruiti V, Donati D, Giraudo G (2020) Feasibility of enhanced recovery after surgery program in colorectal surgery during COVID-19 pandemic in Italy: should we change something?.Updates Surg 72:319-320. https://doi.org/10.1007/s13304-020-00827-1.

34. Zhu D, Wu Q, Lin Q, Wei Y (2020) Modified management mode for colorectal cancer during COVID-19 outbreak - a single-center experience.Aging (Albany NY) 12:7614-

7618.https://doi.org/10.18632/aging.103099.

35. Di Saverio S, Pata F, Gallo G, et al (2020) Coronavirus pandemic and colorectal surgery: practical advice based on the Italian experience.Colorectal Dis. 22:625634.https://doi.org/10.1111/codi.15056.

36. COVIDSurg Collaborative (2020) Mortality and pulmonary complications in patients undergoing surgery with perioperative SARS-CoV-2 infection: an international cohort study.Lancet. 396:27-38. https://doi.org/10.1016/S0140-6736(20)31182-X.

37. Vecchione L, Stintzing S, Pentheroudakis G, et al (2020) ESMO management and treatment adapted recommendations in the COVID-19 era: Colorectal cáncer. ESMO Open. 2020;5. https://doi.org/10.1136/esmoopen-2020-000826. 
Figures

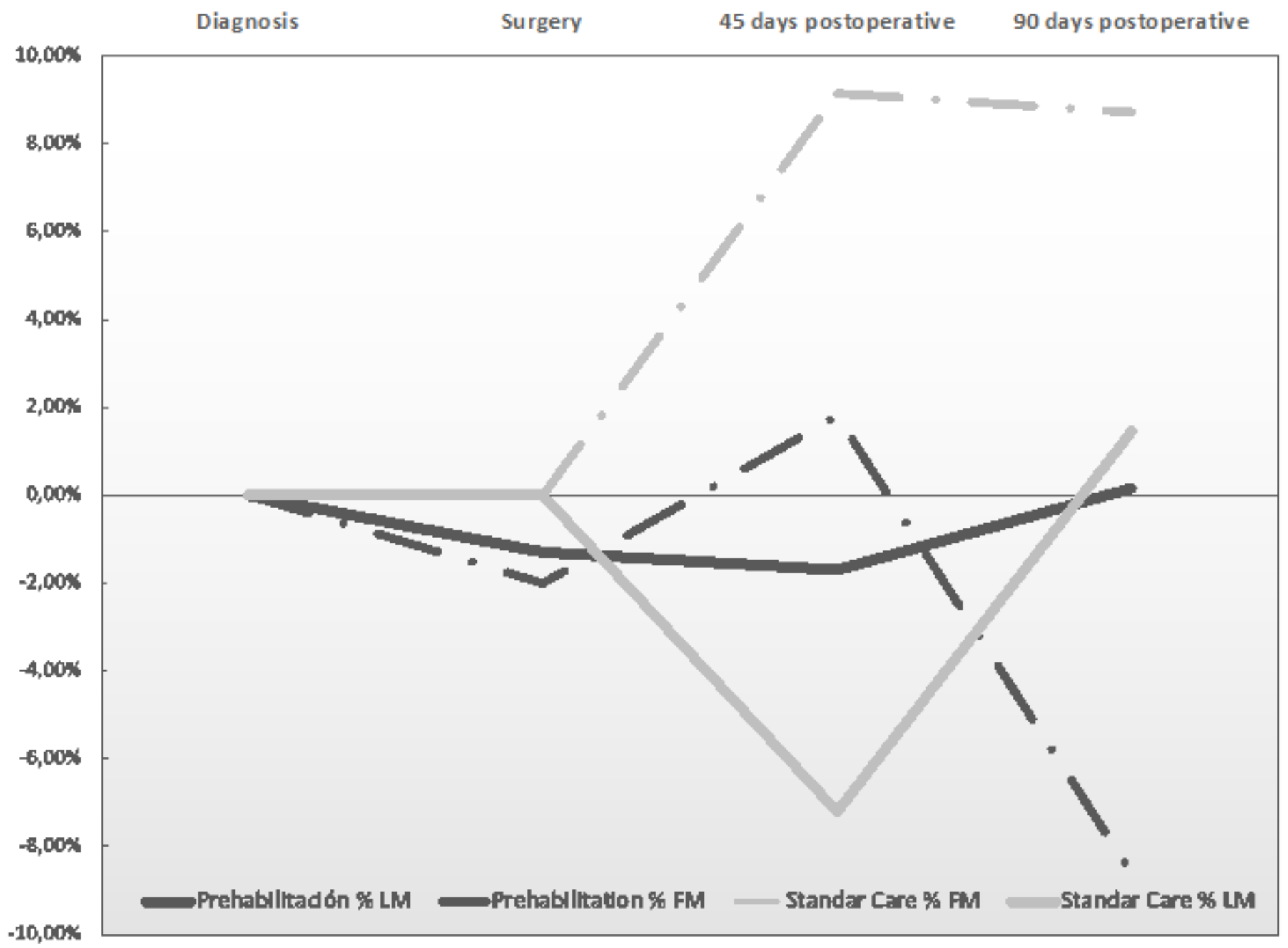

Figure 1

Evolution of changes in LM and FM in the $\mathrm{PH}$ and $\mathrm{SC}$ group since diagnosis. 